\title{
IPEM topical report 2: The first UK survey of dose indices from radiotherapy treatment planning computed tomography scans for adult patients
}

\author{
Anne T. Davis ${ }^{1,2,3}$, James Earley ${ }^{1,4}$, Sue Edyvean ${ }^{5}$, Una Findlay ${ }^{6}$, Rebecca Lindsay ${ }^{1,7}$, Andrew Nisbet ${ }^{1,3,4}$, \\ Antony L. Palmer ${ }^{1,2,3}$, Rosaleen Plaistow ${ }^{1,8}$, Matthew Williams ${ }^{1,9}$, Tim J. Wood ${ }^{1,10,11}$ \\ ${ }^{1}$ Institute of Physics and Engineering in Medicine: Radiotherapy and Diagnostic Radiology Special Interest Groups, \\ Doses to patients from $\mathrm{x}$-ray imaging in radiotherapy working party \\ ${ }^{2}$ Medical Physics Department, Portsmouth Hospitals NHS Trust, Portsmouth, PO6 3LY, UK \\ ${ }^{3}$ Department of Physics, Faculty of Engineering and Physical Science, University of Surrey, Guildford, UK \\ ${ }^{4}$ Department of Medical Physics, Royal Surrey County Hospital NHS Foundation Trust, Guildford, UK \\ ${ }^{5}$ Radiation Dosimetry Department, Centre for Radiation Chemicals and Environmental Hazards, Public Health \\ England, Chilton, Oxon, OX11 0RQ, UK \\ ${ }^{6}$ Medical Exposure Department, Centre for Radiation Chemicals and Environmental Hazards, Public Health \\ England, Chilton, Oxon, OX11 0RQ, UK \\ ${ }^{7}$ Medical Physics Department, St James's Institute for Oncology, Leeds, LS9 7TF, UK \\ ${ }^{8}$ Medical Physics Department, Cambridge University Hospitals NHS Foundation Trust, Cambridge CB2 0QX, UK \\ ${ }^{9}$ Medical Physics, Velindre NHS Trust, Velindre Road, Whitchurch, Cardiff, CF14 2TL, UK \\ ${ }^{10}$ Radiation Physics Department, Queen's Centre for Oncology and Haematology, Castle Hill Hospital, Hull \& East \\ Yorkshire Hospitals NHS Trust, Castle Road, Hull, HU16 5JQ, UK \\ ${ }^{11}$ Faculty of Science, University of Hull, Cottingham Road, Hull, HU6 7RX, UK
}




\section{$1 \quad$ Abstract}

CT scans are an integral component of modern radiotherapy treatments, enabling the accurate localisation of the treatment target and organs-at-risk, and providing the tissue density information required for the calculation of dose in the treatment planning system. For these reasons, it is important to ensure exposures are optimised to give the required clinical image quality with doses that are as low as reasonably achievable. However, there is little guidance in the literature on dose levels in radiotherapy CT imaging either within the UK or internationally.

This IPEM topical report presents the results of the first UK wide survey of dose indices in radiotherapy CT planning scans. Patient dose indices were collected for prostate, gynaecological, breast, 3D-lung, 4D-lung, brain and head/neck scans. Median values per scanner and examination type were calculated and national dose reference levels and 'achievable levels' of CT dose index $\left(\mathrm{CTDI}_{\mathrm{vol}}\right)$, dose-length-product (DLP) and scan length are proposed based on the third quartile and median values of these distributions, respectively.

A total of 68 radiotherapy CT scanners were included in this audit. The proposed dose reference levels for CTDI ${ }_{\text {vol }}$ and DLP are; prostate $16 \mathrm{mGy}$ and $570 \mathrm{mGy} . \mathrm{cm}$, gynaecological $16 \mathrm{mGy}$ and $610 \mathrm{mGy} . \mathrm{cm}$, breast $10 \mathrm{mGy}$ and $390 \mathrm{mGy} . \mathrm{cm}, 3 \mathrm{D}-$ lung $14 \mathrm{mGy}$ and $550 \mathrm{mGy} . \mathrm{cm}, 4 \mathrm{D}-$ lung $63 \mathrm{mGy}$ and $1750 \mathrm{mGy} . \mathrm{cm}$, brain $50 \mathrm{mGy}$ and 1500 mGy.cm and head/neck $49 \mathrm{mGy}$ and $2150 \mathrm{mGy} . \mathrm{cm}$. Significant variations in dose indices were noted, with head/neck and 4D-lung yielding a factor of eighteen difference between the lowest and highest dose scanners. There was also evidence of some clustering in the data by scanner manufacturer, which may be indicative of a lack of local optimisation of individual systems to the clinical task. It is anticipated that providing this data to the UK and wider radiotherapy community will aid the optimisation of treatment planning CT scan protocols.

Keywords: Diagnostic reference levels (DRLs), radiotherapy CT, UK audit 


\section{Introduction}

The use of Computed Tomography (CT) is an integral component of the radiotherapy treatment planning process (Mutic et al 2003, IAEA 2012). The primary function of the planning CT is to accurately map the positions of the treatment target and organs-at-risk and, through use of a treatment planning system (TPS), provide electron or physical density information that can then be used in treatment plan development and dose calculations. Another important factor that needs to be considered in these types of scan is the requirement for the patient to be imaged in the treatment position, which often requires the use of specialised equipment to ensure accurate and reproducible localisation throughout the radiotherapy pathway. These clinical requirements mean that scan protocols used for radiotherapy planning are unique when compared with similar diagnostic CT applications, and often place additional technical limitations on the exposure settings and scanner hardware. For example, it is usually necessary to scan all patients with the same x-ray tube voltage to ensure accurate conversion from CT number (Hounsfield units) to electron density as differences in attenuation induced through changing the beam energy could result in errors in the TPS dose calculations. This fixed tube kilovoltage approach may limit the scope for dose and image quality optimisation of planning CT scans (Davis et al 2017). It is also important to ensure the scanner hardware is compatible with radiotherapy treatment requirements, which will include the need for a flat couch top and may require a large scanner bore to accommodate patients on bulky immobilisation equipment. The increased x-ray tube to detector distance on large bore CT scanners may impact dose and/or image quality. It is for these reasons that almost 20 years ago, the American Association of Physicists in Medicine (AAPM) recommended scan protocols be developed that are specific to the disease site being treated (Fraassa et al 1998). The potential for CT protocol optimisation in radiotherapy has increased since these recommendations were published, with most therapy centres now owning dedicated CT scanners that incorporate many of the features required for planning scans, alongside a number of dose and image quality optimisation features found on diagnostic CT scanners such as automatic exposure control (AEC) with or without automatic tube current modulation (ATCM) and iterative reconstruction (IR). The process of optimisation should ensure that the quality of the CT image is suitable to allow accurate outlining of the treatment target and surrounding organs whilst minimising radiation dose received by the patient (Davis et al 2017). This requirement for optimisation is also a legal requirement in the UK under the Ionising Radiation (Medical Exposure) Regulations 2017 (IRMER 2017).

Diagnostic reference levels (DRLs), as a tool to support optimisation, have been a European legislative requirement for diagnostic imaging procedures since 2000. Recent updates to the regulations have reinforced the requirement for DRLs (EU 2014, IRMER 2017). The principle is that if DRLs for CT examinations are appropriately defined, average dose indices delivered on individual CT scanners for groups of standard size patients should not consistently exceed these reference levels; if they do, an investigation should be undertaken and remedial action implemented where appropriate. For CT, Dose Length Product (DLP) and volume CT dose index (CTDI $\left.{ }_{\mathrm{vol}}\right)\left(\mathrm{Huda}_{\mathrm{C}}\right.$ and Mettler 2011) are the dose metrics of choice. Traditionally DRLs have been set on the basis of national or international multi-centre audit (Shrimpton et al 2014, PiDRL 2016), with recent guidance from the International Commission on Radiological Protection (ICRP 2017) recommending the third quartile of scanner median dose indices for the determination of DRLs (note, previous UK national DRLs (NDRLs) have used the third quartile of 
scanner mean doses (PHE 2016)). An additional aid to optimisation proposed in ICRP Publication 135 is the national median value (also known as the 'achievable dose' (Brink et al 2012, Iball et al 2017)); this promotes a more proactive approach to optimisation to ensure that dose levels are not simply reduced to just meet the national DRL, but to ensure the lowest doses achievable. However, with such quantities it is vital to ensure that image quality remains commensurate with the intended clinical purpose.

Reference levels for radiotherapy planning CT examinations have never been defined in the UK and there are very few publications worldwide which provide dose data. A recent study compared CTDI ${ }_{\mathrm{vol}}$, DLP and scan length for breast planning CT scans only, and included data from six radiotherapy centres in Ireland (50\% of the centres in the country) (Connor et al 2016). Despite the common clinical task, significant variations in exposure parameters were noted and DRLs of $26 \mathrm{mGy}_{\text {for }} \mathrm{CTDI}_{\mathrm{vol}}$ and $732 \mathrm{mGy} . \mathrm{cm}$ for DLP were proposed. The requirement for optimisation of CT protocols for radiotherapy planning was highlighted as an important issue, even from this relatively small sample of six CT scanners. Since CT examinations used for diagnosis have a different clinical purpose and scan length to those acquired for radiotherapy planning, the DRLs published by Public Health England (PHE) may not be suitable as an optimisation tool in radiotherapy CT (Shrimpton et al 2014, Holroyd and Edyvean 2018). This is also true of European and international guidance on DRLs for CT imaging, emphasising the need for robust large scale national dose audits of radiotherapy $\mathrm{CT}$ imaging practice.

This paper presents the results of the first large scale UK audit of CT doses delivered during planning CT scans, with a view to proposing the first UK national reference levels for a range of common clinical sites. The audit has been undertaken by a working party formed by the radiotherapy and diagnostic radiology special interest groups of the Institute of Physics and Engineering in Medicine (IPEM). The working party remit is to investigate current practice, techniques used and doses delivered during imaging for radiotherapy. This publication contains the results of the first phase of this audit. 


\section{Method \\ Examination selection and pre-audit questionnaire}

Seven radiotherapy CT scan protocols were initially selected for the dose audit. Common examinations were chosen to increase the likelihood of collecting a high volume of dose index data. These were: head and neck, brain, breast (no nodes), prostate (no nodes), gynaecological (no nodes), lung 3D and lung 4D. Examinations that covered the nodes were excluded from the audit as it was anticipated that variations in scan length could be significant between patients; by requesting 'no node' examinations, it was felt that practice would be more standardised (and nodal examinations would simply be an extension of these protocols). Associated guidance that was provided for these examinations is given in Table 1.

Table 1: CT protocols and guidance issued with the data collection form.

\begin{tabular}{ll}
\hline CT protocol & Guidance \\
\hline Breast & No nodes \\
Gynaecological & No PA nodes \\
Head and neck & Nasal/oral cancers which include neck nodes \\
Lung 3D & \\
Lung 4D & \\
Brain & Excluding stereotactic planning scans; excluding palliative \\
Prostate & No nodes \\
\hline
\end{tabular}

A pre-audit questionnaire was issued to 73 UK radiotherapy centres, consisting of 63 National Health Service (NHS) centres and 10 private providers. Some of these centres had multiple sites. The purpose of the questionnaire was to collect information on CT scanner types in use in radiotherapy centres, to confirm whether the selected examinations represented the most common CT examinations, to confirm if patient weight information was available, to identify the extent of paediatric imaging/treatment, and to establish whether centres would participate in a national dose audit.

\section{Data collection}

A Microsoft Excel ${ }^{\mathrm{TM}}$ (Washington, USA) data collection form was developed based on previous national dose audits for SPECT CT and PET CT (Iball et al 2017), and C-spine CT (Holroyd and Edyvean 2018). For each examination type the form required information on the CT scanner make, model and age. Scan protocol details requested included tube voltage, tube current, whether AEC was used, whether helical or axial scanning was employed, acquisition field of view, pitch and collimation settings. Parameters relevant to image formation were collected including image slice thickness, reconstruction algorithm, reconstruction field of view, and whether iterative reconstruction was used. Information about other imaging modalities, use of intravenous contrast agent and the accuracy of scanner displayed CTDI $_{\mathrm{vol}}$ compared with measured values was also collected. Centres were asked to provide $\mathrm{CTDI}_{\mathrm{vol}}$, DLP and scan length values for individual patients, ensuring that the data set for each examination included a minimum of 10 , and up to 30 adult patients. Patient weight was requested where available. Dose from CT localiser radiographs was to be excluded where possible. 
Four different instruction sheets were produced showing where to find the relevant data on the different makes of scanner. The aim of this was to make data collection easier for participating centres and to ensure the required data were collected in a consistent manner. The call for data, together with accompanying forms and instructions, was distributed to radiotherapy centres by e-mail using a contact list for heads of Radiotherapy Physics or Medical Physics departments held by the IPEM, the mail-bases for UK Medical Physics and Engineering and the UK CT Users Group, and via a contact list for radiotherapy managers held by The Society and College of Radiographers. Completed data collection forms were received over a period of 3 months from March to May 2017. After that time centres who had submitted data sets with dose indices from fewer than 10 patients were contacted, and allowed another month to provide more data.

\section{Data review and processing}

During the processing phase, data sets from each centre were anonymised and allocated unique identifiers. Any sets with dose indices for fewer than 8 patients were excluded. The reason for this was that patient weight or size has a significant impact on dose where AEC is used and small numbers of patients cannot be representative of the typical dose delivered by a single scanner (Shrimpton et al 2014). Where centres had submitted several scan protocols for a given examination which were clearly for different clinical purposes, these sets were retained as separate data (and given alphabetical subscripts in their unique identifiers to distinguish them). If centres submitted separate data for the same clinical indication, but there was only a minor difference in scan protocol (e.g. with or without contrast, but all other parameters were the same), the data sets were combined into a single pool.

All data were reviewed to check for anomalies, and centres contacted to confirm accuracy where necessary. All processed data were then copied into a custom built Microsoft Access ${ }^{\mathrm{TM}}$ (Washington, USA) database. The database was interrogated with a Matlab ${ }^{\mathrm{TM}}$ (MathWorks, Massachusetts, USA) graphical user interface during analysis. Median and mean values of $\mathrm{CTDI}_{\mathrm{vol}}$, DLP and scan length were calculated for each examination data set per scanner. Where patient weight was provided, the impact from very large and small patients on median and mean values was evaluated. It was noted that when calculating mean dose indices for a particular scanner a large dose from a heavy patient could have a disproportionate effect on the result. Therefore the decision was made to use median values from each scanner data set when processing the data, which is in line with new guidance from the ICRP (ICRP 2017). 95\% confidence intervals were calculated and are shown as error bars on all plots with median values. Scanner mean values are also included to allow for cross referencing against other published values where scanner means have been used (e.g. Connor et al 2016).

From technical information supplied by Toshiba Medical Systems Ltd it was known that scanners running software version 4.63 or earlier, indicated CTDI $_{\mathrm{vol}}$ is the maximum value delivered during a scan, not the mean as is the case on all other systems (Tsalafoutas et al 2012). Conversely, mean CTDI ${ }_{\mathrm{vol}}$ values are used to calculate DLP. Centres with Toshiba scanners were asked to provide the software version so that those reporting maximum CTDI $_{\text {vol }}$ values could be identified and excluded from the calculation of national reference values if tube current modulation was used, since these systems could significantly skew the proposed values. DLP from these scanners were retained as they are calculated in the same way as all other scanner models surveyed. 
For brain scans and head and neck scans, some systems were configured to indicate CTDI $\mathrm{vol}_{\mathrm{values}}$ for a $16 \mathrm{~cm}$ diameter phantom, whilst others gave it for a $32 \mathrm{~cm}$ diameter phantom. For ease of comparison and presentation of results, the data for $\mathrm{CTDI}_{\mathrm{vol}} 32 \mathrm{~cm}$ and DLP $32 \mathrm{~cm}$ were multiplied by a size-specific dose estimate factor of 2.06 (AAPM, 2011) so that they could be compared with the $\mathrm{CTDI}_{\mathrm{vol} 16 \mathrm{~cm}}$ values. For brain scans, this matches the methodology used in another recent UK CT dose audit (Iball et al 2017) and also allows comparison against dose indices for diagnostic quality head CT scans. The same correction factor was applied for head and neck scans for consistency.

National values of median, first and third quartile, minimum and maximum were calculated using the whole data set for each examination type. The third quartile values were rounded to produce recommended national reference levels and median values were rounded to provide 'achievable levels'. To propose national reference levels and 'achievable levels' for any clinical indication, a minimum of 10 data sets (with at least 8 patient's worth of exposure information) from 10 different systems were required. 


\section{Results \\ Pre-audit questionnaire}

A total of 59 centres responded, corresponding to $80 \%$ of the identified UK radiotherapy centres. The responders covered 92 radiotherapy CT scanners of which the percentage share of scanner manufacturers was $24 \%$ from Siemens Healthineers, 23\% from GE Healthcare, 30\% from Philips Healthcare and 23\% from Toshiba Medical Systems Ltd. For each of the suggested examinations, the number of centres confirming that they carried out those scan types was: 54 for head and neck; 44 for brain; 57 for breast (no nodes); 56 for prostate (no nodes); 46 for gynaecological; 53 for lung 3D and 48 for lung 4D. Additionally 9 centres identified abdominal scans as being relatively frequent. Fewer than half the centres said that they routinely recorded patient weight and many do this only for certain sites, for example head and neck so as to monitor patient weight loss during the course of treatment. Only 16 centres (27\%) indicated that they imaged paediatric patients.

After review of the responses the decision was made to include only doses from adults in the main audit but to consider a subsequent, but targeted audit for paediatric patients. Since many centres did not have patient weight available then the collection of patient weight information would be desirable but not mandatory; the abdomen examination was not included in the selected list as the number of centres that identified this as a frequently used protocol was low; and the initial choice of the seven selected examinations was ratified.

\section{Audit of UK radiotherapy treatment planning CT scans and proposed reference levels}

A total of 55 centres submitted data, but one was excluded as sample sizes were insufficient for inclusion in the analysis. From the 54 centres remaining, data was submitted for 68 different CT scanners (see Table 2). The age of the scanners ranged from 1 to 12 years with the mean age being 6.8 years. Typically, each scanner data set contained sufficient data for 6 or 7 types of examination (or variations thereof). The lowest number of full data sets per scanner was one (i.e. suggesting a scanner that may only be used for one type of scan, such as breast), and the highest number was 10 (note, this centre submitted multiple data sets for variations on the given clinical indications e.g. with and without contrast, breath-hold and free-breathing). Centres were also asked to provide information on $\mathrm{CTDI}_{\mathrm{vol}}$ accuracy, with 34 scanners (50\%) giving data that demonstrated a CTDI ${ }_{\mathrm{vol}}$ accuracy range from 0 to 19\%, with the mean being 7\% (note, it was not clear if data was a positive or negative deviation from the displayed value, so only the absolute deviations were considered). These are within typical manufacturer tolerances for this metric, and demonstrate that no system was operating outside normal limits. As data was not available for all systems, no corrections have been applied to the data in this study. A summary of the data collected is presented in Table 3, and details of typical CT scan parameters are given in Table 4; further information related to individual clinical indications are also given over the following sections. It should be noted that not all centres provided $\mathrm{CTDI}_{\mathrm{vol}}$, DLP and scan length data (or in the case of older Toshiba scanners, CTDI $\mathrm{vol}_{\mathrm{vol}}$ values were excluded if they represented the maximum value for a protocol that used tube current modulation), so the sample sizes for each metric in Table 3 are not identical. Table 5 gives the ratios of the maximum-to-minimum and third-to-first quartile scanner median CTDI $_{\mathrm{vol}}$, DLP and scan length values for each examination. This demonstrates the wide variation in practice for nominally the same clinical task. Table 6 presents the proposed UK reference and achievable levels for the seven different adult radiotherapy treatment planning CT scans that were considered in this study. 
Table 2: Number of scanners by manufacturer and model including the number using iterative reconstruction (IR).

\begin{tabular}{lcc}
\hline Scanner manufacturer and model & Number (\%) & Number with IR \\
\hline GE Healthcare & & \\
Lightspeed RT 16 & $10(15)$ & 0 \\
Discovery CT 590 & $5(7)$ & 3
\end{tabular}

\section{Philips Medical}

Brilliance Big Bore

$27(40)$

5

\section{Toshiba Medical Systems Ltd}

Aquilion LB

$14(20)$

1

Siemens Healthineers

\begin{tabular}{llll} 
& Definition AS & $4(6)$ & 1 \\
& Open & $7(10)$ & 1 \\
& Edge & $1(2)$ & 0 \\
\hline Total & & $68(100)$ & 11 \\
\hline
\end{tabular}


Table 3: Volume corrected Computed Tomography Dose Index (CTDI ${ }_{v o l}$ ), Dose-Length Product (DLP) and scan length for the examinations audited using scanner median values. PD is the Phantom Diameter used for the $\mathrm{CTDI}_{\mathrm{vol}}$ value, $\mathrm{N}_{\text {patients }}$ is the total number of patients that data was submitted for, and $\mathrm{N}_{\text {scanners }}$ is the number of scanners in the sample for the given metric. Median, minimum and maximum values are based on the distribution of scanner median values.

\begin{tabular}{|c|c|c|c|c|c|c|c|c|c|c|c|c|c|c|}
\hline \multirow{2}{*}{ Examination } & \multirow{2}{*}{$\begin{array}{c}\text { PD } \\
(\mathbf{c m})\end{array}$} & \multirow{2}{*}{$\mathbf{N}_{\text {patients }}$} & \multicolumn{4}{|c|}{$\mathrm{CTDI}_{\mathrm{vol}}(\mathrm{mGy})^{1}$} & \multicolumn{4}{|c|}{ DLP (mGy.cm) } & \multicolumn{4}{|c|}{ Scan length (mm) } \\
\hline & & & $\mathbf{N}_{\text {scanners }}$ & Median & Min. & Max. & $\mathbf{N}_{\text {scanners }}$ & Median & Min. & Max. & $\mathbf{N}_{\text {scanners }}$ & Median & Min. & Max. \\
\hline Breast & 32 & 1527 & 52 & 7.5 & 2.3 & 20.6 & 62 & 283 & 92 & 763 & 59 & 332 & 248 & 407 \\
\hline Gynaecological & 32 & 711 & 27 & 12.1 & 6.0 & 32.4 & 36 & 510 & 207 & 1431 & 33 & 377 & 303 & 474 \\
\hline Lung 3D & 32 & 953 & 42 & 9.6 & 3.9 & 24.2 & 51 & 410 & 149 & 996 & 49 & 367 & 308 & 454 \\
\hline Lung 4D & 32 & 664 & 39 & 35.6 & 11.6 & 194 & 41 & 1174 & 346 & 6426 & 36 & 326 & 174 & 640 \\
\hline Prostate & 32 & 1278 & 54 & 12.9 & 7.0 & 33.8 & 64 & 419 & 280 & 1319 & 62 & 305 & 160 & 523 \\
\hline \multirow{2}{*}{ Brain } & 16 & 755 & 36 & 39.8 & 19.1 & 91.3 & 41 & 1043 & 179 & 2888 & 38 & 250 & 186 & 420 \\
\hline & 32 & 199 & 9 & 26.7 & 14.4 & 45.8 & 11 & 785 & 102 & 1336 & 11 & 248 & 214 & 422 \\
\hline All brain data ${ }^{2}$ & 16 & 954 & 45 & 41.6 & 19.1 & 94.4 & 52 & 1107 & 179 & 2888 & 52 & 248 & 186 & 422 \\
\hline \multirow[b]{2}{*}{ Head and Neck } & 16 & 265 & 12 & 21.5 & 7.8 & 84.8 & 13 & 990 & 302 & 3291 & 13 & 383 & 312 & 534 \\
\hline & 32 & 807 & 34 & 13.2 & 4.6 & 69.5 & 39 & 525 & 166 & 2470 & 39 & 400 & 290 & 585 \\
\hline All H\&N data ${ }^{2}$ & 16 & 1072 & 46 & 25.5 & 7.8 & 143 & 52 & 1077 & 302 & 5088 & 52 & 398 & 290 & 585 \\
\hline
\end{tabular}

${ }^{1}$ Note, CTDI $_{\mathrm{vol}}$ maximum values from Toshiba CT scanners running software version 4.63 or earlier were excluded.

${ }^{2} \mathrm{CTDI}_{\mathrm{vol}}$ and DLP values for $32 \mathrm{~cm}$ phantom converted to $16 \mathrm{~cm}$ phantom values using conversion factor 2.06. 
Table 4: A summary of the key scan parameters used for the different CT examinations, including tube kilovoltage (kV), use of automatic exposure control (AEC) or fixed tube current (mA), iterative reconstruction (IR), and whether axial or helical scans were performed. Most frequent values (mode) for pitch (where appropriate) and radiation beam width are given, along with the range of image slice widths and scan/reconstruction fields of view (FOV).

\begin{tabular}{|c|c|c|c|c|c|c|c|c|c|c|c|c|}
\hline \multirow{2}{*}{ Examination } & \multirow{2}{*}{$\mathbf{N}_{\text {scanners }}$} & \multirow{2}{*}{$\begin{array}{c}\mathbf{k V} \\
(\text { mode })\end{array}$} & \multicolumn{2}{|c|}{ mA control } & \multirow{2}{*}{ IR } & \multicolumn{3}{|c|}{ Scan mode } & \multirow{2}{*}{$\begin{array}{l}\text { Radiation } \\
\text { beam width } \\
\text { (mode, mm) }\end{array}$} & \multirow{2}{*}{$\begin{array}{l}\text { Image slice } \\
\text { width } \\
\text { (range, mm) }\end{array}$} & \multirow{2}{*}{$\begin{array}{c}\text { Scan FOV } \\
\text { (range, } \mathbf{m m})\end{array}$} & \multirow{2}{*}{$\begin{array}{l}\text { Recon. FOV } \\
\text { (range, mm) }\end{array}$} \\
\hline & & & AEC & Fixed & & Axial & Helical & $\begin{array}{l}\text { Helical pitch } \\
\text { (range, mode) }\end{array}$ & & & & \\
\hline Breast & 62 & 120 & 58 & 4 & 11 & 1 & 61 & $0.688-1.375,0.938$ & 24 & $1.25-5.0$ & $350-820$ & $350-700$ \\
\hline Gynaecological & 36 & 120 & 36 & 0 & 5 & 0 & 36 & $0.690-1.375,0.938$ & 24 & $2.0-5.0$ & $400-820$ & $400-700$ \\
\hline Lung 3D & 51 & 120 & 48 & 3 & 9 & 1 & 50 & $0.562-1.375,0.938$ & 24 & $2.0-5.0$ & $350-820$ & $350-700$ \\
\hline Lung 4D & 41 & 120 & 8 & 33 & 3 & 8 & 33 & Variable & 24 & $1.25-3.0$ & $436-820$ & $360-606$ \\
\hline Prostate & 64 & $120^{(\mathrm{a})}$ & 63 & 1 & 9 & 0 & 64 & $0.688-1.375,0.813$ & 24 & $1.25-3.0$ & $400-820$ & $400-700$ \\
\hline Brain & 52 & $120^{(\mathrm{b})}$ & 21 & 31 & 8 & 3 & 49 & $0.438-1.375,0.938$ & 12 & $1.25-3.0$ & $250-820$ & $250-611$ \\
\hline Head and Neck & 52 & $120^{\text {(c) }}$ & 41 & 11 & 9 & 1 & 51 & $0.438-1.375,0.938$ & 24 & $1.25-3.0$ & $350-820$ & $350-700$ \\
\hline
\end{tabular}

\section{Table footnotes}

(a) Three prostate scan protocols, intended for fiducial markers, used $140 \mathrm{kV}$.

(b) Two brain scan protocols used $140 \mathrm{kV}$.

(c) One head and neck scan protocol used $140 \mathrm{kV}$. 
Table 5: Maximum-to-minimum and third-to-first quartile ratios for median values from each scanner in this study.

\begin{tabular}{|c|c|c|c|c|c|c|}
\hline \multirow[b]{2}{*}{ Examination } & \multicolumn{3}{|c|}{ Maximum-to-minimum ratio } & \multicolumn{3}{|c|}{ Third-to-first quartile ratio } \\
\hline & CTDI $_{\text {vol }}$ & DLP & Scan length & $\mathrm{CTDI}_{\text {vol }}$ & DLP & Scan length \\
\hline Breast & 9.0 & 8.3 & 1.6 & 1.9 & 1.9 & 1.1 \\
\hline Gynaecological & 5.4 & 6.9 & 1.6 & 1.6 & 1.6 & 1.1 \\
\hline Lung 3D & 6.2 & 6.7 & 1.5 & 2.0 & 1.9 & 1.1 \\
\hline Lung 4D & 16.7 & 18.6 & 3.7 & 2.8 & 2.2 & 1.1 \\
\hline Prostate & 4.8 & 4.7 & 3.3 & 1.6 & 1.6 & 1.3 \\
\hline Brain & 4.9 & 16.2 & 2.3 & 1.5 & 1.6 & 1.3 \\
\hline Head and Neck & 18.3 & 16.9 & 2.0 & 2.6 & 2.8 & 1.1 \\
\hline
\end{tabular}

Table 6: Proposed reference and achievable levels in terms of CTDI ${ }_{\mathrm{vol}}$, DLP and scan length for radiotherapy treatment planning CT scans performed in the UK.

\begin{tabular}{|c|c|c|c|c|c|c|c|}
\hline \multirow[b]{2}{*}{ Examination } & \multirow[b]{2}{*}{$\begin{array}{l}\text { PD } \\
(\mathbf{c m})\end{array}$} & \multicolumn{3}{|c|}{ Proposed reference level } & \multicolumn{3}{|c|}{ Achievable level } \\
\hline & & $\begin{array}{c}\text { CTDI }_{\text {vol }} \\
(\mathbf{m G y})\end{array}$ & $\begin{array}{c}\text { DLP } \\
(\text { mGy.cm) }\end{array}$ & $\begin{array}{c}\text { Scan } \\
\text { length } \\
(\mathrm{mm})\end{array}$ & $\begin{array}{l}\text { CTDI }_{\text {vol }} \\
(\mathbf{m G y})\end{array}$ & $\begin{array}{c}\text { DLP } \\
(\mathbf{m G y} . c m)\end{array}$ & $\begin{array}{c}\text { Scan } \\
\text { length } \\
(\mathrm{mm})\end{array}$ \\
\hline Breast & 32 & 10 & 390 & 360 & 8 & 280 & 330 \\
\hline Gynaecological & 32 & 16 & 610 & 400 & 12 & 510 & 380 \\
\hline Lung 3D & 32 & 14 & 550 & 390 & 10 & 410 & 370 \\
\hline Lung 4D & 32 & 63 & 1750 & 340 & 36 & 1170 & 330 \\
\hline Prostate & 32 & 16 & 570 & 340 & 13 & 420 & 310 \\
\hline Brain & 16 & 50 & 1500 & 290 & 42 & 1110 & 250 \\
\hline Head and Neck & 16 & 49 & 2150 & 420 & 26 & 1080 & 400 \\
\hline
\end{tabular}




\section{Breast scans}

A total of 62 scanners provided data on breast treatment planning scans. In the majority of centres, helical scans (61) were acquired with AEC (58). The use of iterative reconstruction was most common in the breast scanning data (11 scanners using it), and the most common image slice thickness was $3.0 \mathrm{~mm}$ (25 scanners). Figure 1 demonstrates the impact of iterative reconstruction, as currently implemented on the scanners in this study, with the systems using these techniques distributed amongst those using filtered back projection i.e. there is no clear evidence that iterative reconstruction is being implemented to reduce patient doses at this stage. However, the limited data available in this study makes it difficult to draw any statistically significant conclusions, and it may be worth further study in follow up surveys when it is expected that more scanners will have this feature implemented. Only 2 centres indicated that they sometimes use IV contrast.

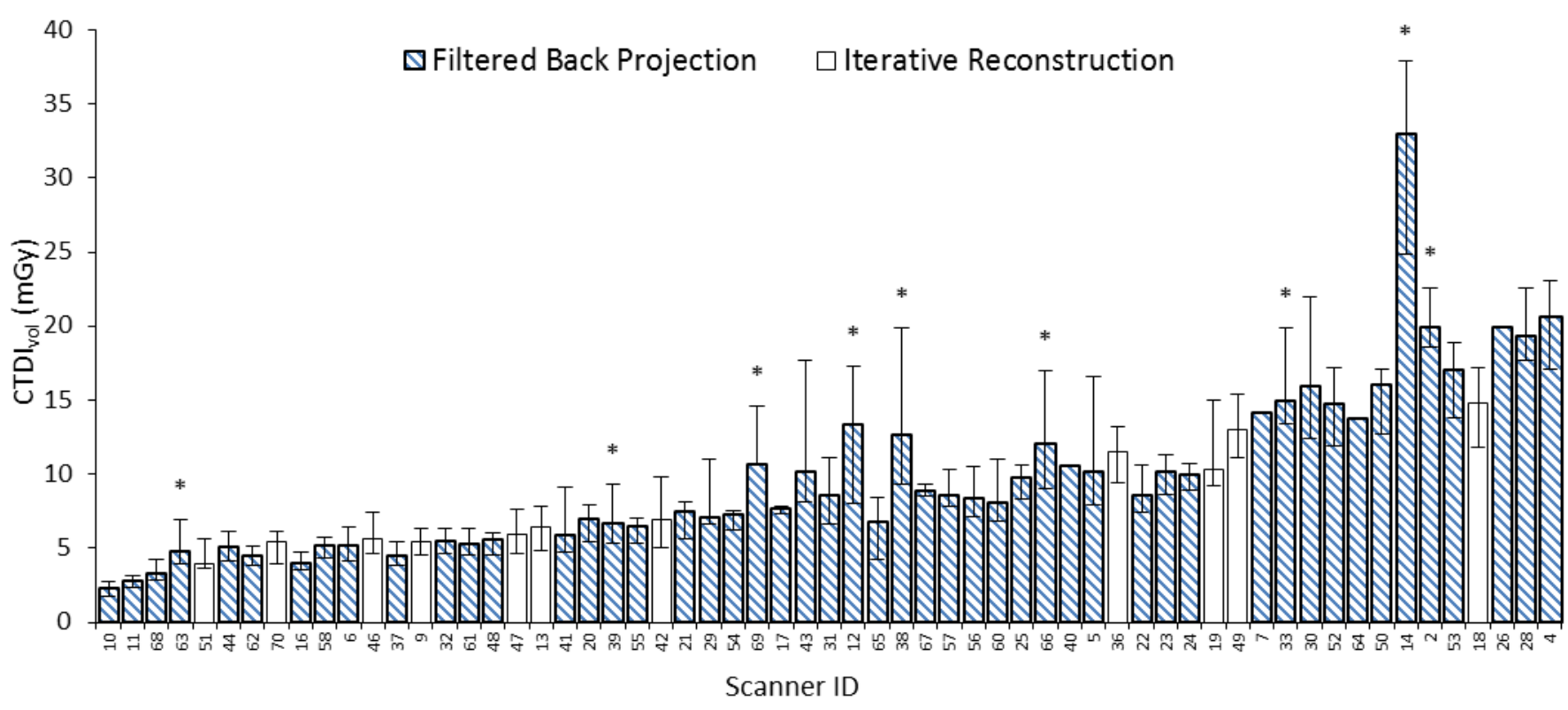

Figure 1: Breast scanning CTDI $_{\mathrm{vol}}$ comparison with filtered back projection or iterative reconstruction indicated for each scanner. Systems are ordered by increasing DLP, and Toshiba CT systems reporting maximum CTDI ${ }_{\mathrm{vol}}$ and using ATCM are indicated $\left(^{*}\right)$. Each bar represents the median scanner value, and error bars are defined by the $95 \%$ confidence intervals.

For comparison with the study by Connor et al (2016), scanner mean values were also used to calculate equivalent reference values for breast CT planning scans (as given in Tables 3 and 6). The achievable (median) CTDI $_{\text {vol }}$ Was $8.4 \mathrm{mGy}$ (range 2.4 to $19.9 \mathrm{mGy}$ ), with a reference value (third quartile) of $12.5 \mathrm{mGy}$. For DLP the achievable dose was $341 \mathrm{mGy} . \mathrm{cm}$ (range 101 to $740 \mathrm{mGy} . \mathrm{cm}$ ), with a reference value of $432 \mathrm{mGy} . \mathrm{cm}$, and for scan length the achievable value was $334 \mathrm{~mm}$ (range 258 to $411 \mathrm{~mm}$ ) with a reference value of $371 \mathrm{~mm}$. Comparing these with the values given in Tables 3 and 6, it is clear that 'average' dose indices are 10-20\% higher when scanner mean is used due to the impact of patient size (with median being more resilient to 'high' doses on large patients in the sample), whilst scan length values are comparable between data sets as these are not exponentially dependent on patient size. 
Five centres provided separate data for standard 'free-breathing' breast scans and deep-inspiration breath hold (DIBH) scans. From this very limited data, 2 centres scanned DIBH patients with the same protocol as for freebreathing, whilst three centres used lower dose DIBH protocols (but same scan range). For this reason, the separate DIBH data sets were not included in this analysis; however, it may be worth inclusion as a separate clinical indication in future dose surveys, to establish if there are any real differences in scan protocols being used, especially as the technique becomes more common. In some centres DIBH were included in the free-breathing set and were indicated in the comments field; these data were included in the analysis as the scans were acquired with the same protocol over the same scan range (but with the patient coached to hold their breath during acquisition).

\section{Gynaecological}

Sufficient data was submitted from 36 scanners, with one centre submitting two sets of data from two different protocols distinguishing between cervical and endometrial scans. All scanners used helical scanning mode and AEC, but only five used iterative reconstruction. One scanner used an image slice thickness of $5 \mathrm{~mm}$, with the rest equally divided between image slice thicknesses of 2.0, 2.5 and $3.0 \mathrm{~mm}$. IV contrast was 'always' used on 10 scanners, and 'sometimes' on a further 19 systems.

Four scanners that used two scan phases are spread throughout the dataset (i.e. not clustered around particular dose values nor are they obvious outliers), and the dose indices are similar to those from other scanners from the same vendors that use similar protocols apart from the number of phases. For two of these four scanners the first phase is a rectal check, before proceeding to the full scan. One of these centres stated the DLP for the rectal check, which typically contributed around 7\% of the total DLP for the 15 (of 20) patients for which this was performed. Another of these four centres stated that an additional contrast scan is sometimes performed, however the number for which this was performed in the submitted dataset is not known.

Lung scans $(3 D \& 4 D)$

Data from 51 scanners were submitted for 3D lung treatment planning. The majority use helical scanning mode (50) and AEC (48). The most common image slice thicknesses were $3.0 \mathrm{~mm}$ (20 scanners) and $2.0 \mathrm{~mm}$ (19 scanners). Nine scanners used iterative reconstruction. Fourteen scanners use IV contrast routinely on 3D lung patients, with a further 24 stating it was used sometimes.

Forty-one scanners submitted data for 4D lung scans. The technique used for acquiring these scans was highly dependent on the manufacturer of the CT system. This has a strong influence on the dose indices for the scan, as demonstrated in Figure 2. It is clear that the protocols and scan technique implemented on the Philips Big Bore tend to yield the lowest CTDI $_{\text {vol }}$ and DLP values, whilst the protocols implemented on Toshiba and GE systems tend to be the highest. Eight of these scanners use axial techniques to acquire the images (all GE), whilst the remaining 33 scanners from the other three vendors acquire slow helical scans. Most protocols used variable parameters that are determined by the breathing rate of individual patients (such as variable pitch on the Philips Big Bore systems). Most 4D scans used fixed mAs techniques (33), with only three scanners using iterative reconstruction. The most 
1

2

3

4

5

6

7

8

10

11

12

13

14

15

16

17

18

19

20

21

22

23

24

25

26

27

28

29

30

31

32

33

34

35

36

37

38

39

40

41

42

43

44

45

46

47

48

49

50

51

52

53

54

55

56

57

58

59

60

common image slice thickness was $3.0 \mathrm{~mm}$ ( 23 scanners). Eighteen scanners use IV contrast routinely, with a further six stating it was used sometimes. 


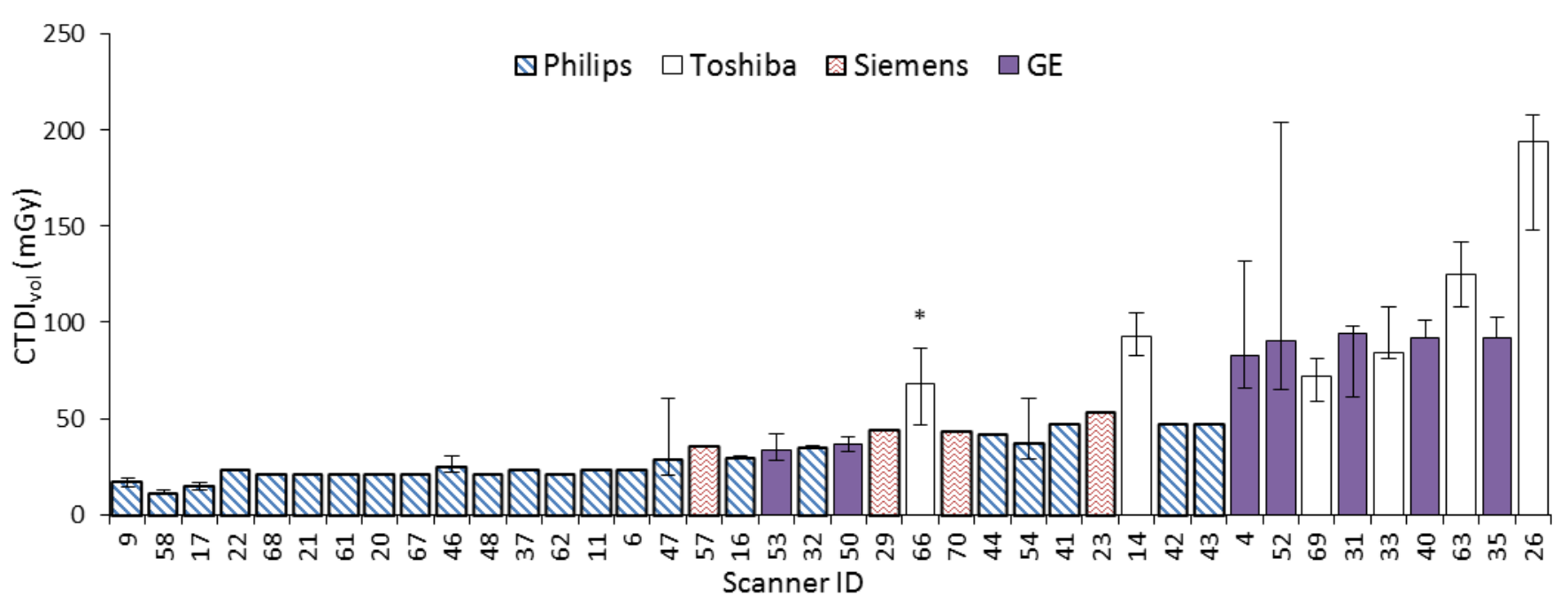

(a)

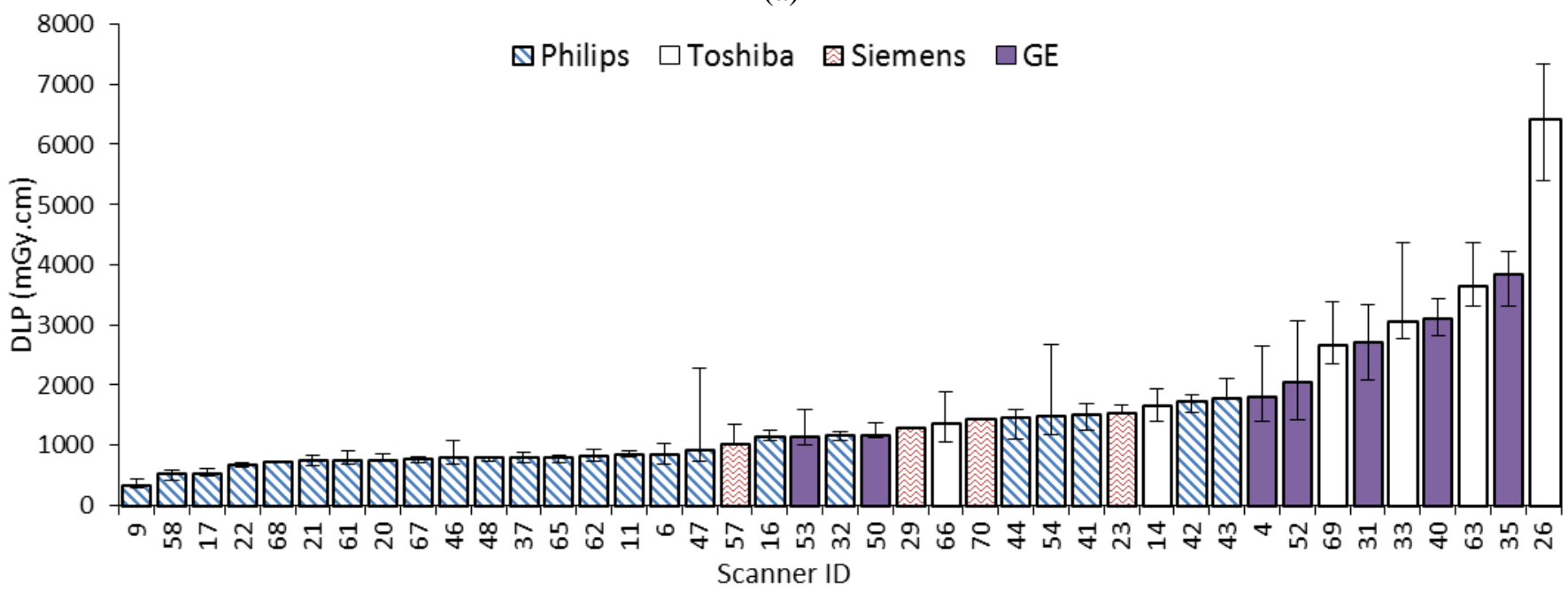

(b)

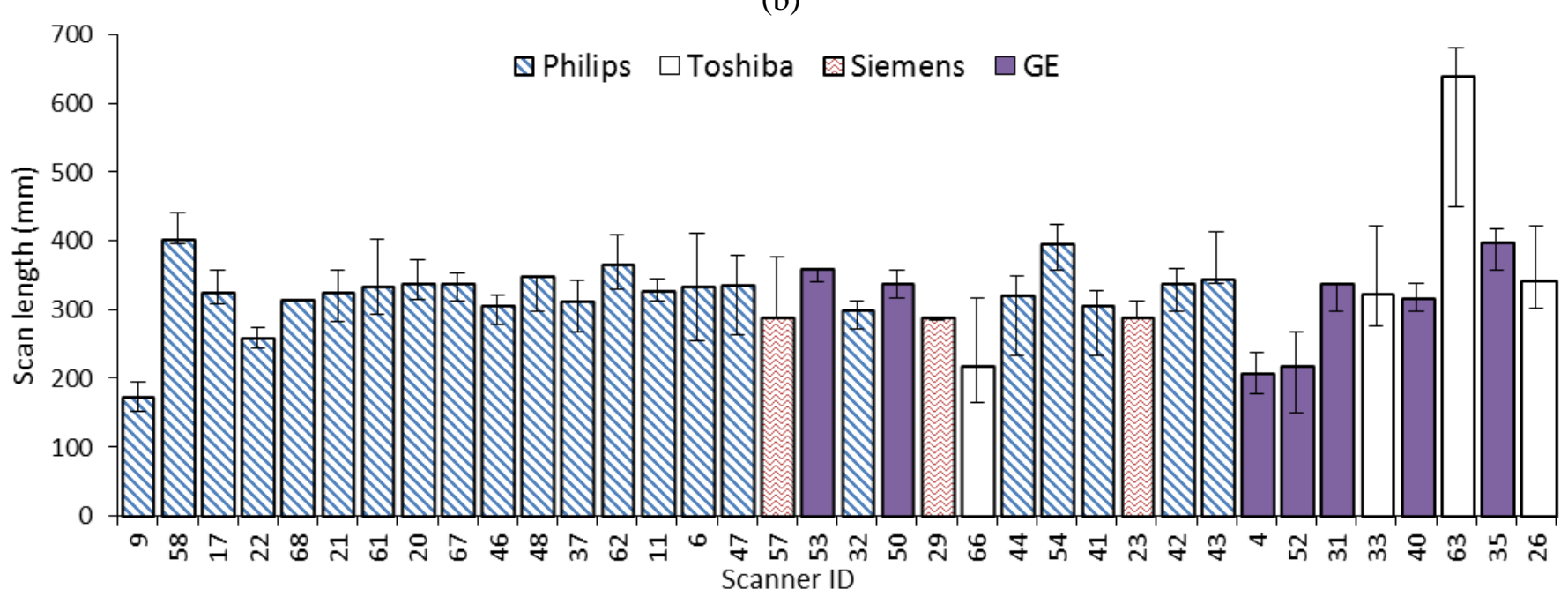

(c)

Figure 2: 4D lung scanning (a) $\mathrm{CTDI}_{\mathrm{vol}}$, (b) DLP and (c) scan length comparisons for the systems included in this study, with vendor indicated for each scanner. Systems are ordered by increasing DLP, and Toshiba CT systems reporting maximum $\mathrm{CTDI}_{\mathrm{vol}}$ and using ATCM are indicated (*). Each bar represents the median scanner value, and error bars are defined by the $95 \%$ confidence intervals. 
In the combined (3D and 4D) lung data sets, seven scanners submitted data for two phase protocols whereby both a 4D and 3D scan were performed as part of the overall treatment planning examination. Figure 3(a-b) shows a comparison of dose indices between the 'standard 3D' scan and those performed as part of the 4D protocol ('3D for 4D'), whilst Figure 3(c) shows a comparison of scan lengths for the standard 3D, 4D and '3D for 4D' scans. Based on this limited data set, it appears that the same protocol is used to acquire the 3D scans in these two examinations (with the exception of one centre that appears to use a lower dose when the 3D scan is part of a 4D study). Out of these seven scanners, it is also apparent that three of these centres use a shorter scan length for the 4D acquisition (and hence, potentially a lower dose), indicating that the 4D imaging tends to focus over the specific regions of interest (e.g. tumour), whilst the 3D scan most likely provides the extended anatomical coverage required for the treatment planning system.

\section{Prostate (no nodes) scans}

There were 64 data sets submitted for prostate scans. Three centres submitted data from two different protocols, with the alternative protocol being for scanning patients with fiducial markers (all set to scan at $140 \mathrm{kV}$ ). Nineteen centres (30\%) stated that a short scan to check the degree of bladder and/or rectum filling was performed prior to the full planning CT; any dose index information provided separately for this check scan was excluded from the results shown in Table 3 (note, this was usually a low dose scan). Just one centre checked the filling of the bladder using ultrasound. The majority of centres did not use IV contrast for prostate scans, though 10 centres indicated that it was used 'sometimes'. In 10 centres MRI was used 'when required', with two centres commenting that MRI was used for patients with hip prostheses. All except one scanner used AEC to set tube current.

\section{Brain scans}

A total of 52 data sets were submitted for brain scans, with 41 scanners giving CTDI $_{\text {vol }}$ and DLP values for a $16 \mathrm{~cm}$ diameter phantom and the remaining 11 scanners indicating a $32 \mathrm{~cm}$ phantom. Fifteen centres routinely use intravenous contrast agent and MRI was used to provide supplementary imaging in 22 centres. One centre submitted two data sets for separate helical and axial scan protocols, but did not provide information as to how the appropriate protocol is selected.

\section{Head and neck scans}

A total of 52 data sets were submitted for head and neck scans, with 13 scanners giving CTDI vol $_{\text {and DLP values }}$ for a $16 \mathrm{~cm}$ diameter phantom and the remaining 39 scanners indicating a $32 \mathrm{~cm}$ phantom. Intravenous contrast agent was used at most centres (43). Three centres submitted separate data sets for contrast and non-contrast scans. For the purposes of data analysis, the contrast and non-contrast data sets were merged for each scanner as all other protocol settings were the same.

Some centres used other forms of imaging to support treatment planning for head and neck patients, though this was not routine on all patients; 17 centres (33\%) indicated MRI images were acquired for some patients, and seven 
centres (14\%) sometimes acquire PET scans. One centre stated that they were using a second reconstruction at smaller field-of-view to assist with outlining. 


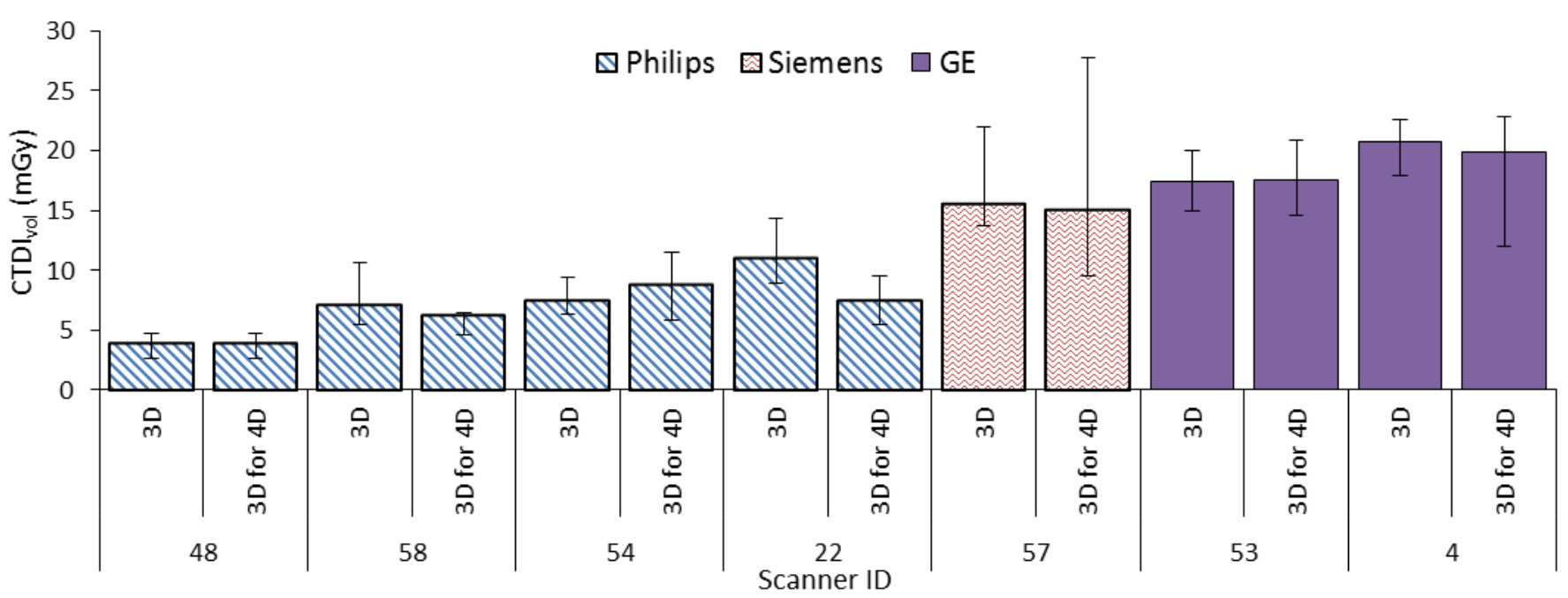

(a)

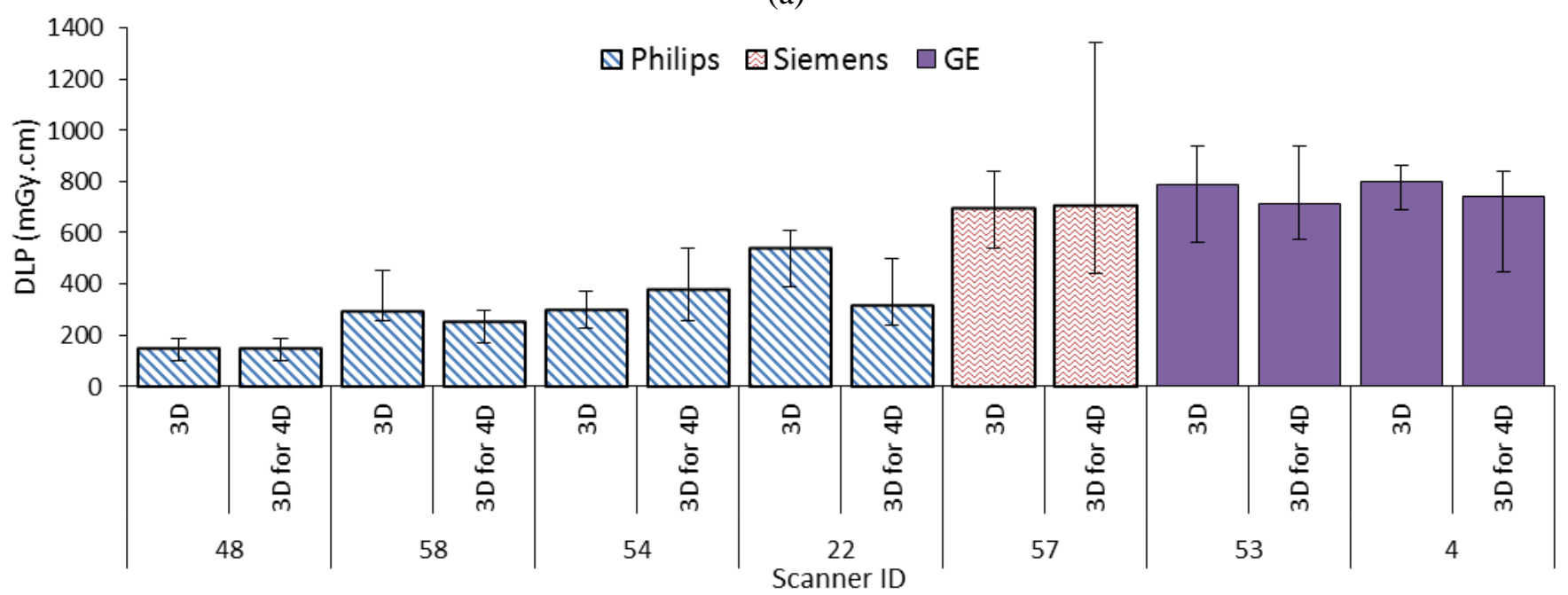

(b)

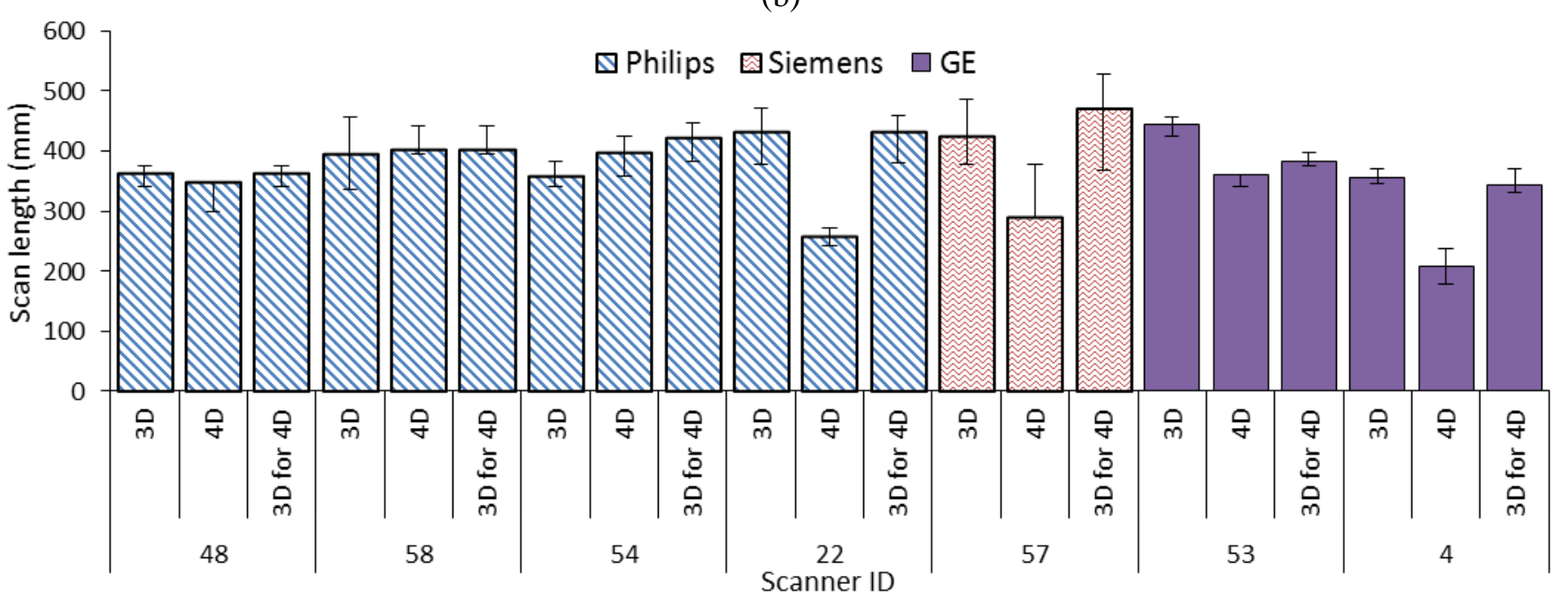

(c)

Figure 3: (a) CTDI $_{\mathrm{vol}}$ and (b) DLP comparisons between 3D scans acquired for lung treatment planning, and those acquired as part of a 4D lung CT examination (note, 4D dose indices are not displayed for clarity as they are approximately three times higher). (c) Scan length comparisons between 3D, 4D and '3D for 4D' scans. Each bar represents the median scanner value, and error bars are defined by the $95 \%$ confidence intervals. 


\section{Automatic exposure control, tube current modulation and patient size}

The use of AEC and tube current modulation is wide spread but not universal across centres and scan protocols. Whilst all centres used AEC for gynaecological scans, several still use fixed tube current for other clinical indications (see Table 4). For head and neck scans, where potentially AEC would help maintain more consistent image quality through the significant variations in patient cross-section, 11 of the 51 centres used fixed tube current. Figure 4 shows how median $\mathrm{CTDI}_{\mathrm{vol}}$ for these scanners is evenly distributed amongst the systems that used AEC. This potentially highlights issues with the optimisation of scan protocols as not only will image quality be highly variable throughout the scan volume (depending on the individual patient cross-section), but there are also significant variations in dose indices due to the configuration of CT protocols; for example, scanners 2 and 38 are the same model of Toshiba scanner (installed within a year of each other at different centres), but gave the lowest and highest $\mathrm{CTDI}_{\mathrm{vol}}$ in the fixed tube current group, due to a factor of three difference in tube current settings (note, neither used iterative reconstruction).

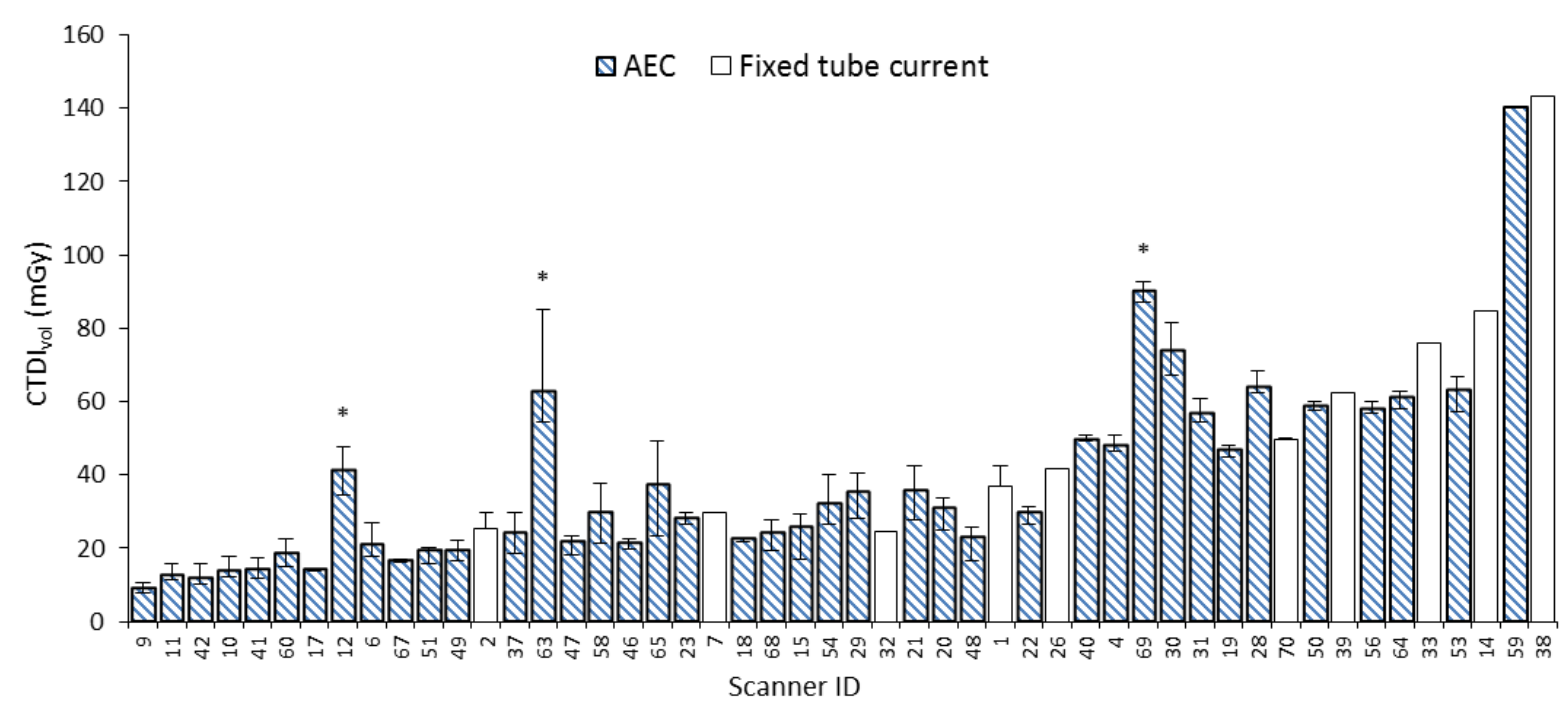

Figure 4: Head and neck CTDI $_{\mathrm{vol}}$ values $(16 \mathrm{~cm}$ phantom) demonstrating the use of AEC or fixed tube current. Systems are ordered by increasing DLP, and Toshiba systems reporting maximum $\mathrm{CTDI}_{\mathrm{vol}}$ and using ATCM are indicated (*). Each bar represents the median scanner value, and error bars are defined by the $95 \%$ confidence intervals.

Another example of the effect of AEC and tube current modulation is shown Figure 5 for 3D lung scans. All but three of the systems use AEC to determine exposure factors (centres 7, 26 and 55 use fixed exposure factors). In this data set, there appears to be some clustering of dose indices from the different manufacturers with the Philips systems towards the lower end of the scale, the GE systems at the upper end, and the Toshiba and Siemens systems spread amongst the middle of the data set. This trend was consistent across most clinical indications in this study. This clustering likely reflects the configuration of default scan protocols, rather than any fundamental differences in technology; with appropriate optimisation, it is expected that such effects would be removed from the data without any detriment to image quality (as evidenced by some scanners breaking this trend e.g. scanner numbers 63 (Toshiba), 23 (Siemens) and 31 (GE) are noticeably lower dose than other similar systems from the same vendor). 


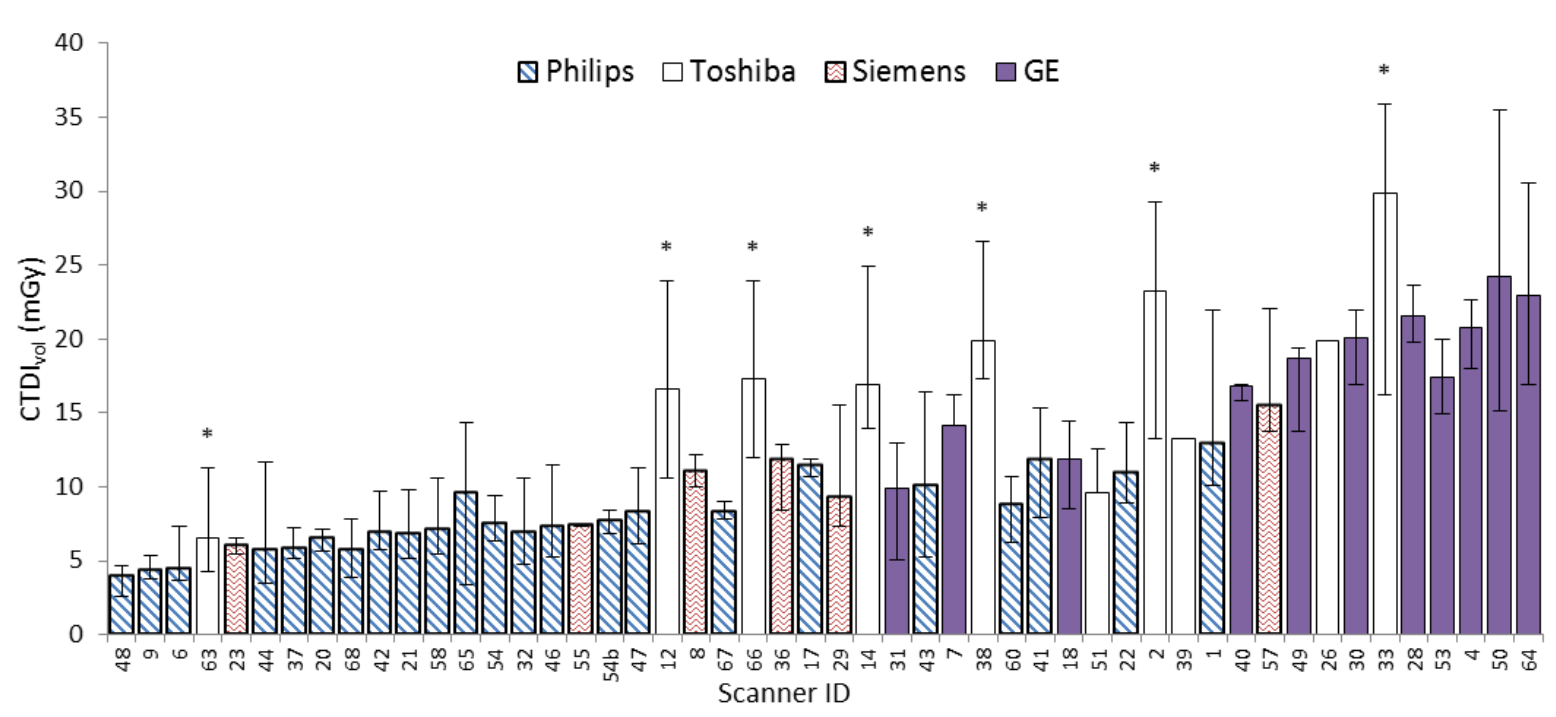

Figure 5: 3D lung scanning $\mathrm{CTDI}_{\mathrm{vol}}$ comparisons for the systems included in this study, with vendor indicated for each scanner. Systems are ordered by increasing DLP, and Toshiba systems reporting maximum CTDI ${ }_{\mathrm{vol}}$ and using ATCM are indicated (*). Each bar represents the median scanner value, and error bars are defined by the $95 \%$ confidence intervals.

Figure 5 also demonstrates the potential impact when trying to use CTDI $_{\mathrm{vol}}$ in dose audits for older Toshiba systems. With the data ordered by increasing DLP (which is always based on the mean CTDI ${ }_{\mathrm{vol}}$ ), the Toshiba scanners that report maximum CTDI $_{\mathrm{vol}}$ become apparent as significant peaks within the data (compared with neighbouring, similar DLP scanners). When comparing scanner median dose indices with the CTDI ${ }_{\mathrm{vol}}$ reference values proposed in this study, it is therefore important to account for such effects; in practice on older Toshiba scanners, it will probably be appropriate to compare with the reference DLP and scan length values only.

Combined with the configuration of the AEC system, typical patient size for the given clinical indication will determine the dose index on each scanner. For prostate CT scans (where both dose index and weight were provided for 25 systems), median CTDI $_{\mathrm{vol}}$ per scanner and individual patient dose indices were compared when plotted against weight (see Figure 6). Patient weight range was large (38 to $179 \mathrm{~kg}$ ) for the sample of 431 patients, and $\mathrm{CTDI}_{\mathrm{vol}}$ values clearly increased with patient size, as would be expected with AEC. When median CTDI ${ }_{\mathrm{vol}}$ and median weight are considered, the average scanner data is more tightly clustered around $80-85 \mathrm{~kg}$, but demonstrates a wide variation in dose due to the configuration of the CT scanner. To illustrate the potential impact of weight on the determination of reference values (note, all data irrespective of whether weight was provided or not was used to determine the reference values given in Table 6), Table 7 shows the results for the subset of data where weight information was provided, compared with the whole data set both in terms of scanner median values (as per ICRP recommendations) and scanner means. From the limited 'with weight' data set, it is clear that the 'typical' patient $(83 \mathrm{~kg})$ for this examination is larger than the historical 'standard' size patient $(70 \mathrm{~kg})$, which is likely reflective of both the changing UK population and the specific clinical indication. This combined with the limited number of patients that can be audited for these types of examination render it impractical to set strict limits on patient weight for inclusion in this audit. Table 7 also demonstrates that patient size has a strong influence on dose index 
reference values when scanner mean values are used, with the DLP reference level $11 \%$ higher than the value obtained with scanner medians in the 'with weight' data set; this is consistent with the results for the breast data. This also demonstrates that using scanner median is a robust technique for dose audit in the absence of patient size information as the two data sets yield reference values within $4 \%$ of each other, whilst using scanner means results in discrepancies of up to $13 \%$. This will be due to scanner median being more resistant to outlier patients who are either very large or very small.

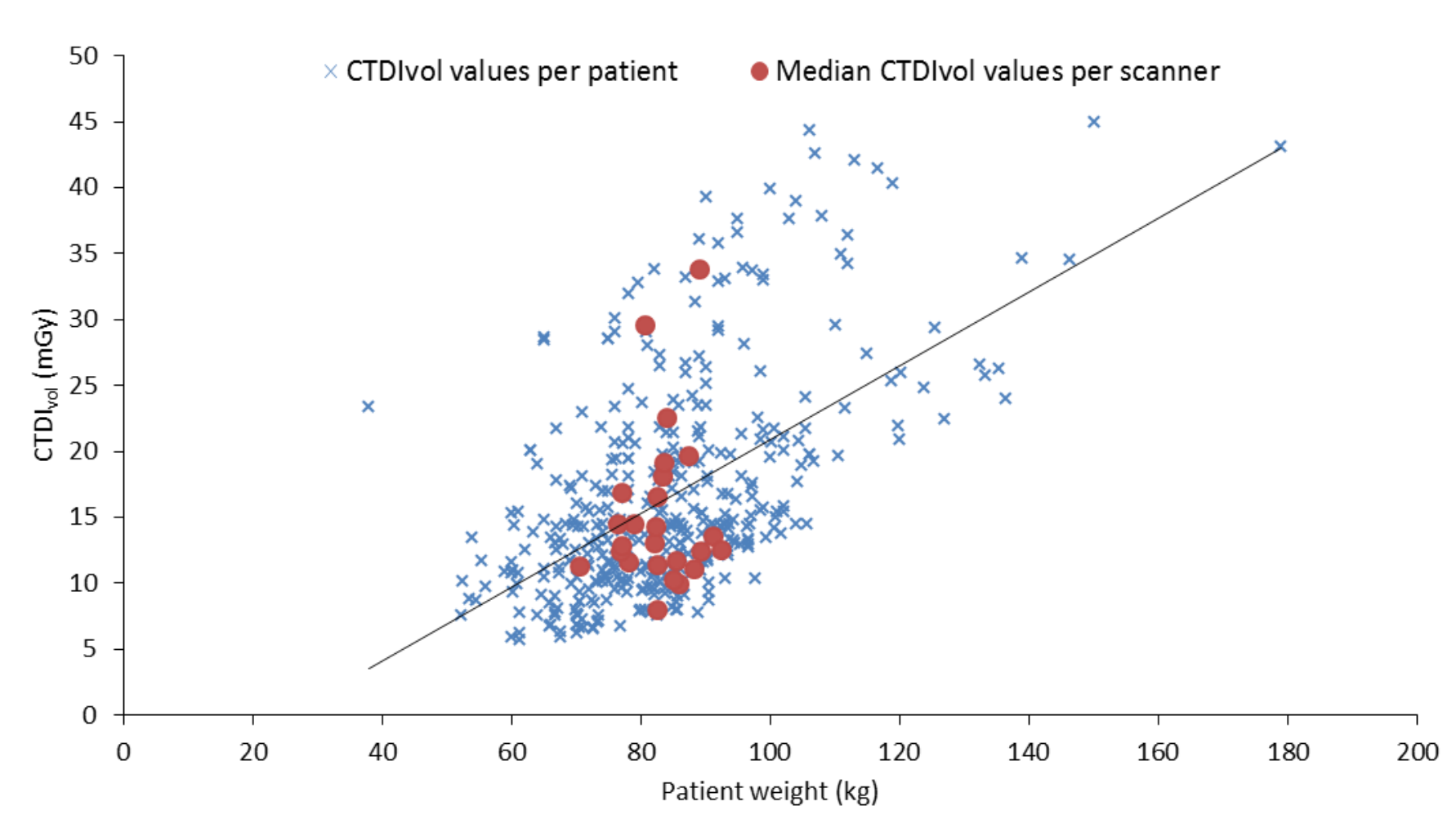

Figure 6: Individual $\mathrm{CTDI}_{\mathrm{vol}}$ versus patient weight for the sub-set of prostate examination data where weight was provided compared with scanner median $\mathrm{CTDI}_{\mathrm{vol}}$ plotted as a function of scanner median weight. A linear fit to the individual patient data is shown to guide the eye.

Table 7: A comparison of prostate reference levels determined with and without patients' weight information using scanner median and mean values.

\begin{tabular}{|c|c|c|c|c|c|c|c|}
\hline \multirow{3}{*}{ Data set } & \multirow{3}{*}{$\mathbf{N}_{\text {scanners }}$} & \multicolumn{4}{|c|}{ CTDIvol (mGy) } & \multirow{2}{*}{\multicolumn{2}{|c|}{ Weight (kg) }} \\
\hline & & \multicolumn{2}{|c|}{ 'Scanner median' data } & \multicolumn{2}{|c|}{ 'Scanner mean' data } & & \\
\hline & & Median & $\begin{array}{c}\text { Third } \\
\text { quartile }\end{array}$ & Median & $\begin{array}{c}\text { Third } \\
\text { quartile }\end{array}$ & Median & $\begin{array}{c}\text { Third } \\
\text { quartile }\end{array}$ \\
\hline 'With weight' only & 25 & 13.1 & 16.9 & 13.8 & 18.8 & 83 & 86 \\
\hline All data & 54 & 12.9 & 16.2 & 13.5 & 16.7 & - & - \\
\hline
\end{tabular}




\section{Discussion}

The wide range of $\mathrm{CTDI}_{\mathrm{vol}}$, DLP and scan length values shows that there is clearly scope to optimise radiotherapy CT scan protocols in the UK. Head and neck and 4D lung scans show the greatest variation between centres with the highest dose scanner delivering approximately 18 times that seen on the lowest $\left(\mathrm{CTDI}_{\mathrm{vol}}\right.$ or DLP). For examinations showing the least variation in dose indices between scanners, namely prostate, gynaecological and 3D lung, the maximum to minimum factor is closer to five. For these scan types AEC usage is more prevalent and appears to be effective at reducing dose index variability. It is also apparent that the central $50 \%$ of data appears to be less variable with ratios between the $3^{\text {rd }}$ and $1^{\text {st }}$ quartile taking values of between 1.5 and 2.0 for all exams with the exception of head and neck and 4D lung, which can be up to a factor of 2.8. The reduced variability in the central $50 \%$ of scanners may be indicative of centres that are potential outliers; this could either be due to the scanners being configured to use either particularly 'high dose' settings, or very low dose settings where the acceptability of image quality may become more of a concern.

Based on the results of this audit, there is some evidence of scanners supplied by a given vendor operating on similar scan protocols, suggesting default protocols are in use without local adjustment and optimisation. This is demonstrated through the clustering of dose index values in Figures 2 and 5, but was also observed in other examination types. Dose indices for 4D lung scans were seen to have particularly strong manufacturer dependence which will be related to the different scan techniques used by each vendor to generate these image sets. However, there are several cases where centres have clearly optimised scan protocols locally as they do not lie within the usual cluster for that vendor; this indicates that the scope for optimisation is not purely defined by the make and model of scanner installed in the department, and relatively simple changes to scan protocols may enable real patient benefits (be it through reduced patient dose or more optimal image quality). It has also been noted that although $16 \%$ of scanners have iterative reconstruction available, the range of dose indices delivered by scanners using this technology currently matches that where filtered back-projection is used. Hence, there is currently no evidence of notable dose reduction resulting from the use of iterative reconstruction in radiotherapy planning CT scans.

The wide spread of dose indices across the sampled data, the clustering by vendor and the variability in implementation of new technology clearly justifies the need for national reference levels to be set as a tool to aid scan protocol optimisation. Dose index $\left(\mathrm{CTDI}_{\mathrm{vol}}\right.$ and DLP) and scan length reference levels based on the third quartile of the distributions of scanner median values have been proposed (like DRLs in diagnostic practice), alongside achievable levels (based on the median of the scanner median distributions). It is hoped that by making use of both of these values, it will be possible to improve CT practice in radiotherapy departments across the UK by not only promoting 'compliance' with the reference levels, but by also utilising the achievable values as a further benchmark. For example, it may be anticipated that the oldest CT equipment should be able to comply with the reference levels but may struggle to match the achievable level, whilst the newest technology should be able to yield dose indices at, or below, the achievable standard. Nevertheless, as is the case with all optimisation work, it is 
important to ensure that compliance with these reference levels does not come at the expense of acceptable image quality for the clinical task at hand. However, given the range of data and the similarity of the many systems in this study, it is unlikely that image quality and reference level compliance would represent a problem for the equipment in UK radiotherapy centres. It is hoped that by making this data available, future surveys of radiotherapy CT planning scans will reveal much less variation in practice across the UK, and better optimisation of the technology available on modern CT scanners. Future surveys may also expand the range of protocols to include new techniques such as deep-inspiration breath-hold, and there may be a need to develop reference levels for Stereotactic Ablative Radiotherapy (SABR) planning scans, where thinner slices may be required for planning purposes (which in turn will impact on image quality).

Another factor that contributes to the level of dose observed on each scanner is the variability in patient size, which in turn may depend on the clinical indication. Traditionally, national dose audits have specified that only patients falling within a particular 'standard' weight range (typically 50 to $90 \mathrm{~kg}$ ) be included, though more recent reviews have taken the pragmatic view that this is no longer required provided sample size is greater than 10 patients per system (Hart et al 2012, Shrimpton et al 2014). Inclusion of patients based on 'standard weight' can prove problematic where, as evidenced by the pre-audit questionnaire, many centres do not routinely record this information or have it readily available at the time of data collection. Other alternatives such as patient crosssectional area may be useful indicators of patient size for routine dose audit, but are difficult to implement in practice due to the time required to make such measurements and the difficulty in defining exactly where in the patient scan volume this should be evaluated. It is also true in the case of radiotherapy planning scans that the clinical condition may have a significant impact on 'typical' patient size, yielding patient groups that do not fit within the 'standard' patient range that has been used historically for diagnostic imaging patient dose audits.

As patient weight information was not available for all systems in this audit, the impact of patient size was investigated on a more limited set of data. Where AEC was used, $\mathrm{CTDI}_{\mathrm{vol}}$ clearly increases with increasing patient weight, and it has been demonstrated that data points at the very low or very high end of the weight and dose index range can skew the mean values for any given scanner. Median values are less affected by this, and have been shown to be a more robust metric for the purposes of dose audit for these types of examination; for this reason the median values per scanner were used to define the proposed reference levels in this study. Whilst this differs from some previous national UK dose audits carried out for diagnostic imaging procedures (Shrimpton et al 2014), it is in line with recently published international guidance (ICRP, 2017) and is effective in the absence of weight information. It has also been a consideration in the latest national audit for C-Spine CT dose indices, where similar trends to those reported in this study have been observed (Holroyd and Edyvean 2018). It is important for centres that wish to compare their local dose indices against the recommended reference values that the sample size for local dose data is sufficiently large, and it is recommended that median values are used for any comparisons. 
For the reference levels proposed in this work and given in Table 6, there are very few radiotherapy CT reference levels published elsewhere that can be used for comparison (either from the UK or internationally). For breast, the 2016 audit of six Irish radiotherapy centres proposed national DRLS of $26 \mathrm{mGy}$ and $732 \mathrm{mGy} . \mathrm{cm}$ for CTDI $\mathrm{vol}_{\text {and }}$ DLP, respectively, using the third quartile of scanner means. Irrespective of whether scanner mean or median values are compared with this data, the UK reference values proposed in this study are approximately 40-60\% lower.

Table 8 gives a comparison of the proposed radiotherapy planning CT reference levels with the closest equivalent diagnostic CT examination UK national DRL. For prostate scans, the proposed reference level of $16 \mathrm{mGy}$ for CTDI $_{\mathrm{vol}}$ compares well with the diagnostic CT NDRLs of $14 \mathrm{mGy}$ for abdomen and $15 \mathrm{mGy}$ for abdomen and pelvis. It should be noted, however, that the UK NDRL values for diagnostic CT scans will be for a different clinical task. As might be expected, gynaecological scans have similar proposed reference levels to those for prostate, with slightly longer scan lengths as may be expected due to the relative size of the structures that are of clinical interest.

Table 8: A comparison of radiotherapy planning CT scan reference levels with the closest equivalent diagnostic CT examination UK national DRL (Shrimpton et al 2014). DLP comparisons should be treated with caution as scan lengths may be quite different between these clinical applications.

\begin{tabular}{|c|c|c|c|c|c|}
\hline \multicolumn{3}{|c|}{ Proposed radiotherapy planning scan reference level } & \multicolumn{3}{|c|}{ Closest 'equivalent' diagnostic CT UK NDRL } \\
\hline Examination & $\mathrm{CTDI}_{\mathrm{vol}}(\mathrm{mGy})$ & DLP (mGy.cm) & Examination & $\mathrm{CTDI}_{\mathrm{vol}}(\mathrm{mGy})$ & DLP (mGy.cm) \\
\hline Breast & 10 & 390 & $\begin{array}{l}\text { Chest } \\
\text { (lung cancer) }\end{array}$ & 12 & 610 \\
\hline Gynaecological & 16 & 610 & $\begin{array}{l}\text { Abdomen \& pelvis } \\
\text { (abscess) }\end{array}$ & 15 & 745 \\
\hline Lung 3D & 14 & 550 & $\begin{array}{l}\text { Chest } \\
\text { (lung cancer) }\end{array}$ & 12 & 610 \\
\hline Lung 4D & 63 & 1750 & - & - & - \\
\hline Prostate & 16 & 570 & $\begin{array}{l}\text { Abdomen \& pelvis } \\
\text { (abscess) }\end{array}$ & 15 & 745 \\
\hline Brain & 50 & 1500 & $\begin{array}{l}\text { Head } \\
\text { (acute stroke) }\end{array}$ & 60 & 970 \\
\hline Head and Neck & 49 & 2150 & $\begin{array}{l}\text { Head } \\
\text { (acute stroke) }\end{array}$ & 60 & 970 \\
\hline
\end{tabular}


With regards to scans of the head, the current UK national diagnostic reference level for brain imaging is $60 \mathrm{mGy}$

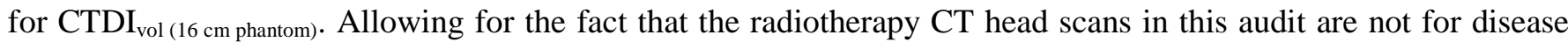
diagnosis, the proposed $\mathrm{CTDI}_{\mathrm{vol}}$ reference levels of $50 \mathrm{mGy}$ for a radiotherapy CT brain scan and $49 \mathrm{mGy}$ for head and neck scans would appear to be appropriate. The DLP reference levels for radiotherapy head scans are notably higher than the diagnostic NDRL of 970 mGy.cm, but this is to be expected due to the longer CT scan length required for the treatment planning process. For 3D lung, the proposed reference level of CTDI $\mathrm{vol}_{\mathrm{v}}$ of $14 \mathrm{mGy}$ is slightly higher than the NDRL of $12 \mathrm{mGy}$ for diagnostic CT scans. In this case, there may be scope for extensive optimisation of practice to reduce doses in radiotherapy, but this must not be at the expense of appropriate image quality for treatment planning.

Some technical challenges related to the CT scanners were identified during this audit. Firstly, older Toshiba scanners which, when AEC is in use, indicate maximum CTDI $_{\mathrm{vol}}$ values, as opposed to the more typical mean CTDI $_{\mathrm{vol}}$ from a patient scan. These systems had to be identified and removed from the CTDI $\mathrm{vol}_{\mathrm{vol}}$ data when determining reference values. These scanners can indicate considerably higher $\mathrm{CTDI}_{\mathrm{vol}}$ values than other scanners when scanning body regions where there is high variation in tissue density or thickness. This is also an important consideration when comparing scanner performance against the reference values proposed in this study; if a centre has an older generation Toshiba CT scanner, it may not be appropriate to compare median CTDI $_{\mathrm{vol}}$ from local audits with the proposed national reference values. However, a comparison with the DLP and scan length reference values would be appropriate and allow optimisation of scan protocols, where necessary. Secondly, the lack of standardisation for phantom size used when displaying CTDI $_{\mathrm{vol}}$ and DLP for scans of the head region is unhelpful and is a potential source of confusion. This was evidenced by one of the submitted sets of data where a correction had been incorrectly applied. Whilst the $32 \mathrm{~cm}$ phantom data collected during this audit has been converted to data for a $16 \mathrm{~cm}$ phantom by multiplying by a standardised figure of 2.06 , the true correction factor used should be measured on each scanner since the conversion factor depends on scanner design. Published data (AAPM 2011) shows that this value can vary from 1.9 to 2.4 for different scanner types. The use of $32 \mathrm{~cm}$ phantom size on some scanners will primarily relate to the use of the large scan field of view as part of the treatment planning process. It is recommended, though, that scanners be set for head and neck and brain imaging, to show $\mathrm{CTDI}_{\mathrm{vol}}$ for a $16 \mathrm{~cm}$ phantom, or at the very least ensure appropriate conversions are performed before comparing with reference values. Both these points highlight the clear need for local operators to very clearly understand the technical features of their scanner.

The information provided by centres on aspects related to imaging practice rather than scan protocol settings provided some interesting information and suggests that there is scope for sharing of good practice. Examples include one centre which is using a 'no dose' ultrasound option to review bladder filling, and $30 \%$ of other centres which undertake a low dose $\mathrm{CT}$ check scan instead prior to performing a prostate $\mathrm{CT}$ scan (which may reduce the number of repeat planning scans performed due to incorrect patient preparation). Some centres appear to be reducing the high dose delivered during 4D-lung scans by minimising scan length where supplementary data exists from 3D scanning of the extended anatomy. 
With regards to the potential limitations of this study, variation in the source of the scan length information is a possible source of error in the results as the method for acquiring this information was not prescribed. The information available is dependent on the manufacturer and model, and the amount of over-scan along the patient axis (required for interpolation in helical scans) can vary between scanners; this may or may not have been included in the quoted scan length submitted to this audit. It is believed that many centres used the coordinates of the first and last images to infer the imaged scan length. There was also no correction applied to any of the datasets for displayed $\mathrm{CTDI}_{\mathrm{vol}}$ accuracy as this information was only provided for $50 \%$ of scanners. However, the mean reported discrepancy was low (7\%), and the maximum (19\%) was within normal manufacturer tolerances for this metric, and so there were no significant concerns about the quality of data submitted. Finally, no image quality data was collected from scanners contributing to this audit. In the pre-audit questionnaire a high proportion of centres expressed interest in participating in a follow-up study, specifically involving image quality assessment; it is therefore hoped that reference image quality metrics may be developed in the future to use alongside these dose index and scan length reference levels for the purposes of optimisation.

\section{Conclusions}

The first UK wide audit of dose indices for adult patients undergoing CT scans for radiotherapy planning has been completed. Reference values and achievable levels for CTDI $_{\mathrm{vol}}$, DLP and scan length have been proposed for seven common types of CT scan. Doses delivered by different scanners varied by a factor of up to 18 times for some scan types, indicating the need for reference dose index values and the possible need for scan protocol optimisation in some UK radiotherapy centres. Scan protocol adjustments to change dose indices should always be accompanied by a rigorous quality assessment to ensure that the images remain suitable for radiotherapy planning.

\section{Acknowledgements}

The authors would like to thank all centres who contributed data to this study, and the Institute of Physics and Engineering in Medicine, and specifically the Radiotherapy and Diagnostic Radiology Special Interest Groups for sponsoring and supporting this working party. 


\section{References}

Boone J M, Strauss K J, Cody D D, McCollough C H, McNitt-Gray M F, Toth T L., 2011. Size specific dose estimates (SSDE) in pediatric and adult body CT examinations (Task Group 204). College Park, MD: American Association of Physicists in Medicine.

Brink J A, Boone J M, Feinstein K A, Michalski J M, Pizzutiello Jr R J, Spelic D C, White S C, Yee J, 2012. Report No. 172 - Reference Levels and Achievable Doses in Medical and Dental Imaging: Recommendations for the United States. [online] Available at: http://www.ncrppublications.org/Reports/172 [Accessed 12/12/17].

Connor SO, Mc Ardle O, Mullaney L, 2016. Establishment of national diagnostic reference levels for breast cancer CT protocols in radiation therapy. Br J Radiol 89: 20160428.

Davis AT, Palmer AL, Nisbet A, 2017. Can CT scan protocols used for radiotherapy treatment planning be adjusted to optimize image quality and patient dose? A systematic review. Br J Radiol 90: 20160406.

EU 2014, Council Directive 2013/59/Euratom of 5 December 2013 laying down basic safety standards for protection against the dangers arising from exposure to ionising radiation. Off J Eur Commun, No. L13.

EuroSafe, 2016. PiDRL European Guidelines on DRLs for Paediatric Imaging. [online] Available at: http://www.eurosafeimaging.org/wp/wp-content/uploads/2014/02/European-Guidelines-on-DRLs-for-PaediatricImaging_Revised_18-July-2016_clean.pdf [Accessed July 2017].

Fraassa B, Doppke K, Hunt M, Kutcher G, Starkschall G, Stern R, Van Dyke J, 1998. American Association of Physicists in Medicine Radiation Therapy Committee Task Group 53: Quality assurance for clinical radiotherapy treatment planning. Med Phys: 25: 10, 1773-1829.

Hart D, Hillier MC, Shrimpton PC, 2012. HPA-CRCE-034. Doses to patients from radiographic and fluoroscopic x-ray imaging procedures in the UK - 2010 Review. Didcot, UK: Public Health England.

Holroyd J R, Edyvean S, 2018. Doses from cervical spine computed tomography (CT) examinations in the UK. Br J Radiol 10.1259/bjr.20170834.

Huda W, Mettler F, 2011. Volume CT Dose Index and Dose-Length Product displayed during CT: What good are they? Radiology: 258: 1, 236-242.

IAEA, 2012. Human Health Series, No. 19, Quality Assurance Programme for Computed Tomography: Diagnostic and Therapy Applications. Vienna, Austria: International Atomic Energy Agency.

Iball GR, Bebbington NA, Burniston M, Edyvean S, Fraser L, Julyan P, Parkar N, Wood T, 2017. A national survey of computed tomography doses in hybrid PET-CT and SPECT-CT examinations in the UK. Nucl Med Commun 38: 6, 459-470.

IRMER 2017. Statutory Instruments No. 1322. The Ionising Radiation (Medical Exposure) Regulations 2017. London: HMSO. 
Mutic S, Palta J R, Butker E K, Das I J, Hug M S, Loo L N, Salter B J, McCollough C H, Van Dyk J. 2003. Quality assurance for computed-tomography simulators and the computed tomography-simulation process: Report of the AAPM Radiation Therapy Committee Task Group No. 66. Med Phys; 30; (10) 2762-2792.

Public Health England (PHE) 2016. National Diagnostic Reference levels. [online] Available at: https://www.gov.uk/government/publications/diagnostic-radiology-national-diagnostic-reference-levels-ndrls. [Accessed July 2017].

Shrimpton PC, Hillier MC, Meeson S, Golding SJ, 2014. PHE-CRCE-013. Doses from Computed Tomography (CT) Examinations in the UK - 2011 Review. Didcot, UK: Public Health England.

Tsalafoutas IA, Thalassinou S, Efstathopoulos EP, 2012. A comprehensive method for calculating patient effective dose and other dosimetric quantities from CT DICOM images. AJR; 199; 133-141.

Vaño E, Miller D L, Martin C J, Rehani M M, Kang K, Rosenstein M, Ortiz-Lopez P, Mattsson S, Padovani R, Rogers A, 2017. Diagnostic reference levels in medical imaging. ICRP Publication 135. Ann. ICRP 46(1). 